\title{
Performance Evaluation of a MIMO-Assisted MPR-MAC over Lossy Channels
}

\author{
Sanaz Barghi, Student Member, IEEE, Hamid Jafarkhani, Fellow, IEEE, \\ and Homayoun Yousefi'zadeh, Senior Member, IEEE
}

\begin{abstract}
Coping with collisions is one of the biggest challenges in the design of MAC algorithms for wireless networks. Recent advances in MIMO communications have provided the possibility of simple detection of colliding packets. In this paper, we introduce a new MIMO/MPR-aware cross-layer MAC/PHY design that is capable of combating collisions through the use of a multiple packet reception technique. Analytical and simulation results show that the proposed MAC design can considerably improve the throughput of a WLAN operating over lossy links.
\end{abstract}

Index Terms-MPR, MIMO, PHY, MAC, Cross-Layer Design, IEEE 802.11.

\section{INTRODUCTION}

A $\mathrm{S}$ the IEEE 802.11 [1] family of standards is gaining popularity among other competing standards, it also faces an increasing demand for high speed wireless communications. When the number of users increases, the inefficiency of the collision avoidance approach holds the IEEE 802.11 MAC algorithm back from serving the users effectively.

Multi-packet reception (MPR) techniques have been suggested as a solution to the above problem. The MPR techniques can greatly improve the throughput of the wireless networks in high traffic/contender conditions [2], [3]. The simple structure of the MIMO-MPR receivers and their reliable performance make them viable candidates for the future wireless communication systems. The V-BLAST technique [4] facilitates detection of up to $N$ independent streams of data using an $N$-antenna receiver. By exploiting space-time coding, the array processing techniques of [5] and [6] provide diversity in addition to offering simple multi-packet detection algorithms.

In [7], the authors have introduced a multi-packet reception MAC/PHY algorithm for ad-hoc networks which utilizes VBLAST. In [8], a joint MPR-MAC and adaptive resource allocation algorithm is proposed for MIMO WLANs. Moreover, a combination of V-BLAST and MIMO array processing is used in [9] to implement a multi-user MIMO system. Under the assumption of transmitting over an error free transmission channel, the authors of [2] study a cross-layer MAC algo-

Manuscript received February 17, 2010; revised August 17, 2010 and November 3, 2010; accepted November 5, 2010. The associate editor coordinating the review of this letter and approving it for publication was S. Bhashyam.

The authors are with the Center for Pervasive Communications and Computing (CPCC), University of California, Irvine (e-mail: \{sbarghi, hamidj, hyousefi\}@uci.edu).

This work appears in part in the Proceedings of IEEE ICC 2010. It was supported in part by a research contract from the Boeing Company.

Digital Object Identifier 10.1109/TWC.2010.120310.100240 rithm for WLANs with single antenna terminals and multiple antenna access points (APs).

In this paper, we first propose a cross-layer MAC/PHY MPR algorithm based on the interference cancellation technique of [5]. Next, we study the performance of our algorithm in a WLAN network operating over lossy channels. Our algorithm differs from that of [2] in the MPR technique it employs. Unlike the work of [2], we also consider the bit error rate (BER) associated with transmission over wireless channels in our analysis and simulation. Our results highlight the importance of the choice made for the MPR technique in the PHY and how a poor choice may even lower the network throughput instead of improving it. Our simulation and analysis results illustrate the significant improvement in the rate of packet delivery as the result of leveraging our proposed MPR technique.

The organization of the paper is as follows. In Section II and III, we discuss the MPR-PHY and the MPR-MAC algorithms, respectively. Section IV presents the saturation throughput analysis of a WLAN leveraging the introduced MPR-MAC/PHY algorithm. We provide our numerical results in Section V illustrating the throughput gains of our MPR technique over traditional IEEE 802.11 and the method of [2]. Conclusions are provided in Section VI.

\section{Multiple Packet Reception}

The focus of this work is on the detection of up to two simultaneously transmitted packets. All discussions can be easily extended to the detection of more than two simultaneously transmitted packets. We also note that our discussion revolves around a typical random topology of nodes utilizing an AP and forming a WLAN. We assume all nodes including the AP are equipped with two antennas.

The MPR-MAC algorithm will be discussed in detail in the next section. Briefly speaking, the MPR-MAC algorithm relies on a sequence of RTS/CTS/DATA/ACK messages as in IEEE 802.11 standard [1]. RTS and DATA packets are sent from user terminals, CTS and ACK messages are the corresponding responses from the AP. If only one of the active terminals transmits over the channel, the physical layer applies the single packet detection algorithms. Two simultaneously transmitted RTS/DATA packets are detected by MPR methods.

Multiple RTS packets are detected differently from multiple DATA packets. Terminals transmit their BPSK modulated RTS messages using one antenna. When more than one RTS packets arrive at the AP, the AP detects the overlapping RTS packets by either ILSP or ILSE [10] blind iterative algorithms. 
These algorithms solve maximum-likelihood estimator's minimization problem by iteratively improving their estimate of the channel matrix and signal vectors. Sign ambiguity can be resolved using the known parts of the RTS message.

The AP uses its two antennas and Alamouti coding [11] to transmit CTS and ACK messages. Therefore, terminals will use Alamouti decoders to detect CTS and ACK packets.

In [2], the authors suggest transmitting DATA packets over one antenna and separating $M$ packets using a minimum mean square error (MMSE) detector at an $(N \geq M)$-antenna receiver. This method provides a diversity order of $N-M-1$ for each packet. For the case of 2-antenna AP, single-packet detection provides a diversity order of 2 and double-packet detection provides a diversity order of 1 for each packet. A lower diversity order translates into a higher BER, and consequently a higher packet drop rate. Our proposal is to use two antennas for transmitting Alamouti-coded DATA packets and an array processing technique [5], [6] at the receiver to detect two simultaneously transmitted packets. If the terminals and the AP have two antennas, this MPR method provides a diversity order of 2 for each packet.

A failed transmission introduces a price measured in terms of wasted transmission time. The price of a collision is an RTS transmission time plus the timeout for an RTS packet. When a DATA packet transmission fails because of bit errors, the price is RTS+CTS+DATA transmission time plus the timeout for a DATA packet. An MPR method with a high BER may waste the channel time for a longer period without delivering any packets in comparison to the time wasted for the collision of two RTS packets. Hence, to improve the BER performance of MPR methods, terminals use binary $\mathrm{BCH}$ channel codes [12] to code DATA packets. An exhaustive search over different code rates in different SNRs determines the required code rate for the target packet error rate (PER). While we choose $\mathrm{BCH}$ to improve the BER performance of the MPR method, it is important to note that it is possible to utilize any other error correcting code scheme.

Compared to single-packet detection, MPR requires additional effort for estimating the channel coefficients and keeping transmissions synchronized. As for any other coherent detection method, any error in channel estimation leads to a higher BER for our proposed MPR method. In addition, our system is sensitive to the loss of synchronization just like many other MPR methods. Finally, a packet drop in the PHY of an MPR system lowers the final throughput of the associated MAC.

\section{MPR-MAC ALGORITHM}

In this section, a MAC algorithm for a system capable of detecting 1 or 2 simultaneously transmitted packets at physical layer is introduced. The MAC algorithm is designed as an extension of IEEE 802.11 standard with RTS/CTS signaling. The goal is to solve the scalability problem of IEEE 802.11. Therefore, our MPR-MAC algorithm inherits some of the shortcomings of IEEE 802.11 MAC such as its fairness problem in distributed networks. Identical to IEEE 802.11 standard, the signaling sequence follows the RTS/CTS/DATA/ACK pattern. CTS and ACK messages will have an additional receiver address (RA) field of 6 Bytes long in order to accommodate the second terminal's address in the case of MPR. Note that for this MPR network, collisions happen when more than two RTS packets reach the AP. Two simultaneously transmitted RTS packets initiate transmission of one CTS packet. In case of collision, i.e., transmission of more than two simultaneous RTS packets, all transmitters increase their backoff window sizes and wait for the next random time to retransmit their requests.

When two RTS packets are detected correctly by the PHY layer, the AP fills the RA fields in the CTS message with the corresponding terminals' addresses. Otherwise, the globally known AP address will be written to the second RA field. Upon detection of the CTS message at the terminals, terminals immediately know whether they are the only user transmitting over the channel or if they are to share the channel with another terminal. If the two RA fields of the CTS message are filled with terminals' addresses, terminals will fragment their packets and code the fragments with rate $R$ for DATA packet transmission. Depending on whose address is placed in which RA field, the corresponding terminals decide which one of the two sets of predefined training sequences to use.

When the receiver detects DATA packet(s), RA fields of the ACK message are filled similar to those of the CTS message. After a successful transmission of the ACK packet, the current transmission is terminated.

The overhead of using the array processing MPR method is the extra receiver address field added to CTS and ACK packets. In addition to this overhead, the single antenna MPR method adds a coding overhead in the case of double packet transmission.

Besides collisions, bit errors can cause a transmission to fail over lossy channels. The latter represents another case in which the packet should be scheduled for retransmission. Bit errors may affect any of the RTS/CTS/DATA/ACK messages. In the next section, lossy channels are considered and the throughput of the introduced MPR-MAC algorithm is calculated.

\section{THROUGHPUT ANALYSIS}

In this section, we calculate the saturation throughput of a WLAN utilizing double-packet detector MAC in the absence of hidden terminals. By saturation throughput, we mean that all terminals always have data ready to be transmitted. For the saturation throughput of an MPR network, our calculations are based on the virtual slot definition proposed in [13], [14]. A virtual slot is the time interval between two consecutive decrements of a backoff counter for a terminal. Decrements of the backoff counter may be separated by an idle time slot when the channel is free or a complete transmission interval when the channel is busy. The probability of transmission in a virtual slot, $\tau$, is given by the following equation [13]:

$$
\tau=\frac{2(1-2 p)}{(1-2 p)(W+1)+p W\left(1-(2 p)^{m}\right)}
$$

where, $p$ is the conditional probability of the failure, $W$ is the minimum backoff window size, and $m$ is the number of backoff stages. 
To keep calculations simple and tractable we assume that the fragment burst process is turned off; only one fragment is transmitted for MPR DATA transmission. This is equivalent to send one shorter packet with redundancy instead of the original packet. The assumption is valid for saturation throughput analysis.

Packet transmission may fail because of either collision or bit errors. Therefore, the conditional probability of failure, $p$, is given by:

$$
p=P_{c o l}+P_{e 1} \cdot P_{1}+P_{e 2} \cdot P_{2}
$$

In Eq. (2), $P_{c o l}$ is the conditional collision probability. Further, the products $P_{e 1} \cdot P_{1}$ and $P_{e 2} \cdot P_{2}$ are conditional error probabilities in the cases of single and double transmission, respectively. The individual probabilities are calculated by the following equations:

$$
\begin{aligned}
P_{c o l} & =1-(1-\tau)^{n-1}-(n-1) \tau(1-\tau)^{n-2} \\
P_{1} & =(1-\tau)^{n-1} \\
P_{2} & =(n-1) \tau(1-\tau)^{n-2} \\
P_{e 1} & =1-P_{R 1} P_{C}^{2} P_{D 1} \\
P_{e 2} & =1-P_{R 2}^{2} P_{D 2} P_{C}^{2}-P_{R 2} P_{C}^{2} P_{D 1}\left(1-P_{R 2}+P_{R 2}\left(1-P_{C}\right)\right)
\end{aligned}
$$

In the equations above, $n$ is the number of active terminals. Further, $P_{R 1}, P_{C}, P_{D 1}$, and $P_{A}$ are probabilities of receiving RTS, CTS, DATA, and ACK packets correctly when using the single packet detection method, respectively. The quantities $P_{R 2}$ and $P_{D 2}$ represent the probabilities of the correct detection of RTS and DATA packets, respectively, when the MPR method is employed. By solving Eqs. (1) and (2) relating $\tau$ and $p$, we can identify the probability of transmission in each virtual slot, $\tau$, for a given backoff window size and a given number of active terminals.

For an MPR network, there are nine types of virtual slots with durations indicated below:

1) Idle slot: $T_{1}=\delta$

2) Collision slot: $T_{2}=R T S+\sigma+E I F S+\delta$

3) RTS error slot: $T_{3}=R T S+\sigma+E I F S+\delta$

4) CTS error slot: $T_{4}=R T S+\sigma+S I F S+C T S+\sigma+$ $E I F S+\delta$

5) Data error slot: $T_{5}=R T S+\sigma+S I F S+C T S+\sigma+$ $S I F S+D A T A+\sigma+E I F S+\delta$

6) ACK error slot: $T_{6}=R T S+\sigma+S I F S+C T S+\sigma+$ $S I F S+D A T A+\sigma+S I F S+A C K+\sigma+E I F S+\delta$

7) Successful single transmission slot: $T_{7}=R T S+\sigma+$ $S I F S+C T S+\sigma+S I F S+D A T A+\sigma+S I F S+$ $A C K+\sigma+D I F S+\delta$

8) One successful delivery in a double transmission slot: $T_{8}=T_{7}$

9) Two successful delivery in a double transmission slot: $T_{9}=T_{7}$

For the above items, $\sigma, \delta, S I F S, D I F S$ and EIFS represent the one way propagation delay, a slot duration, short interframe space, DCF inter-frame space, and extended inter-frame space as specified by IEEE 802.11 , respectively. In addition, the required times to transmit the corresponding packets are $R T S, C T S, D A T A$, and $A C K$. Table I lists the parameters used in this paper to obtain numerical and simulation results
TABLE I

THE TABLE OF USED PARAMETERS BASED ON DSSS PHY SPECIFICATION OF IEEE 802.11 STANDARD.

\begin{tabular}{|l|c|}
\hline Propagation Delay & $1 \mu \mathrm{s}$ \\
\hline SIFS & $10 \mu \mathrm{s}$ \\
\hline SlotTime & $20 \mu \mathrm{s}$ \\
\hline DIFS & SIFS+2SlotTime \\
\hline MAC header $(\mathrm{H})$ & 272 bits \\
\hline PHY header & 192 bits \\
\hline RTS & $160+$ MAC header bits \\
\hline CTS & $122+(48)+$ MAC header bits \\
\hline ACK & $122+(48)+$ MAC header bits \\
\hline EIFS & SIFS+CTS (ACK)+DIFS \\
\hline
\end{tabular}

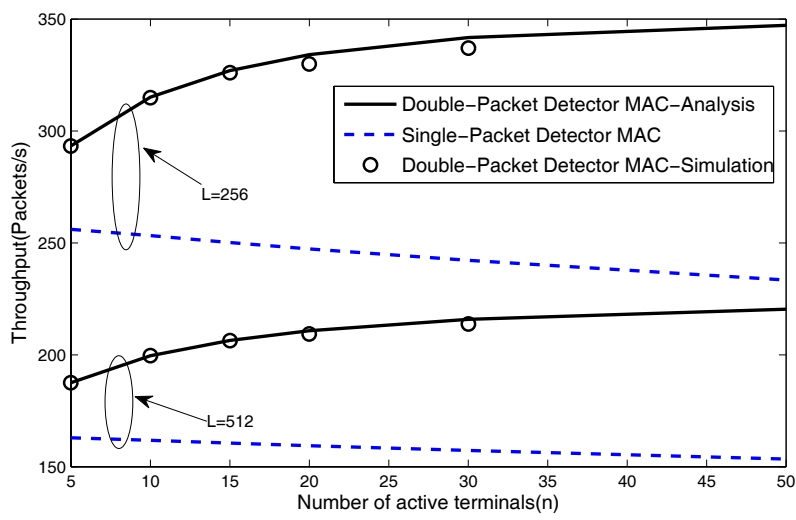

(a)

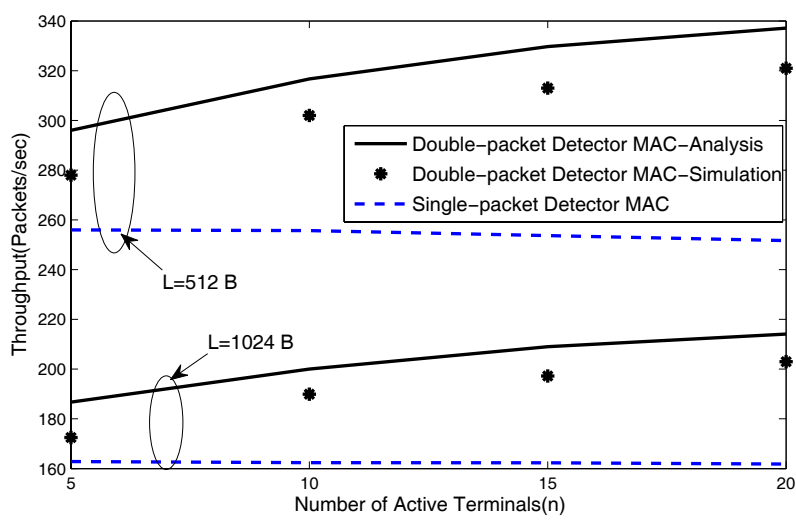

(b)

Fig. 1. A throughput comparison of the double-packet detector MAC algorithm and IEEE 802.11 for a different number of active nodes utilizing (a) a fixed BER simulated PHY model with a data rate of $1 \mathrm{Mbps}$, and (b) a Rayleigh fading simulated PHY model with a data rate of 2 Mbps. While the fixed parameters are set as $W=32$ and $m=3, L$ represents the length of MSDU in Bytes.

based on those of the direct sequence spread spectrum (DSSS) PHY specifications in the IEEE 802.11 standard.

To identify the probability of each time slot, we calculate the four probabilities below:

$$
\begin{aligned}
P_{\text {idle }} & =(1-\tau)^{n} \\
P_{t r} & =1-(1-\tau)^{n} \\
P_{s 1} & =\frac{n \tau(1-\tau)^{n-1}}{P_{t r}}=\frac{n \tau(1-\tau)^{n-1}}{1-(1-\tau)^{n}}
\end{aligned}
$$




$$
P_{s 2}=\frac{\left(\begin{array}{c}
n \\
2
\end{array}\right) \tau^{2}(1-\tau)^{n-2}}{P_{t r}}=\frac{\left(\begin{array}{c}
n \\
2
\end{array}\right) \tau^{2}(1-\tau)^{n-2}}{1-(1-\tau)^{n}}
$$

In the equations above, $P_{\text {idle }}$ and $P_{t r}$ are the probabilities of having an empty virtual slot and having a transmission, respectively. Further, $P_{s 1}$ and $P_{s 2}$ represent the probabilities of having a single transmission and having double transmissions without collision both conditioned on having at least one transmission.

Denoting $P_{k}$ the probability of having a virtual slot type $k$, the values of $P_{k}$ for $k \in\{1, \cdots, 9\}$ are given by (4).

If we define throughput as the number of bits per second delivered to the AP in the MAC-layer frame body, i.e., MAC service data unit (MSDU), the throughput of the MPR-MAC over lossy channels is given by:

$$
S=\frac{\left(P_{7} L_{1}+P_{8} L_{2}+2 \times P_{9} L_{2}\right)}{\sum_{i=1}^{8} P_{i} T_{i}}
$$

where $L_{1}$ is the number of bits in the MSDU when channel coding is not used and $L_{2}$ is the number of data bits in the MSDU when channel coding is used for MPR. Therefore, for transmission of packets with constant length, the relationship $R\left(L_{1}+H\right)=L_{2}+H$ holds where $R$ is the rate of the channel coding and $H$ is the length of the MAC-layer header. The throughput of IEEE 802.11 can be easily calculated from the same set of equations by setting $P_{c o l}=1-(1-\tau)^{n-1}$ and $P_{e 2}=P_{s 2}=0$.

\section{NUMERICAL RESULTS}

To investigate the performance of the MPR/IEEE 802.11 MAC over lossy channels, we use the analysis from the previous section. For a signal to noise ratio (SNR) of $20 \mathrm{~dB}$ and a target $P E R$ of 0.05 , performing an exhaustive search reveals an optimal coding rate $R$ of 0.6 for the single antenna scenario of [2]. No channel coding is required for doubleantenna nodes using the array processing MPR in the same setting.

The curves in Fig. 1 represent the throughput of the singlepacket detector MAC and the array processing MPR-MAC using the array processing method for MSDU lengths of $256 \mathrm{~B}$ and $512 \mathrm{~B}$. In order to validate the analytical results, $N S 2$ is used to simulate a WLAN of one AP and $n$ nodes all using our proposed double-packet detector MAC. The IEEE 802.11 module of $N S 2$ is modified to support the doublepacket detector MAC. Since NS2 simulator does not directly implement the PHY-layer and our MAC module is aware of the multi-packet reception, we use two different approaches for implementing a packet drop event. First, we consider a fixed BER the value of which is determined solely as a function of SNR. Fig. 2 illustrates the results of a MATLAB simulation of the MPR method used for this first approach. Second, we implement a packet drop event by generating Rayleigh fading channel coefficients for each packet in NS2 and use the equivalent SNR calculations of [15] to represent that packet's instantaneous packet drop probability. For analysis, we use the average PER calculated by the MATLAB simulations of the MPR method. We use a constant bit rate (CBR) traffic pattern in our NS2 experiments. Each simulation point in the graph represents the average of 5 independent runs of a traffic

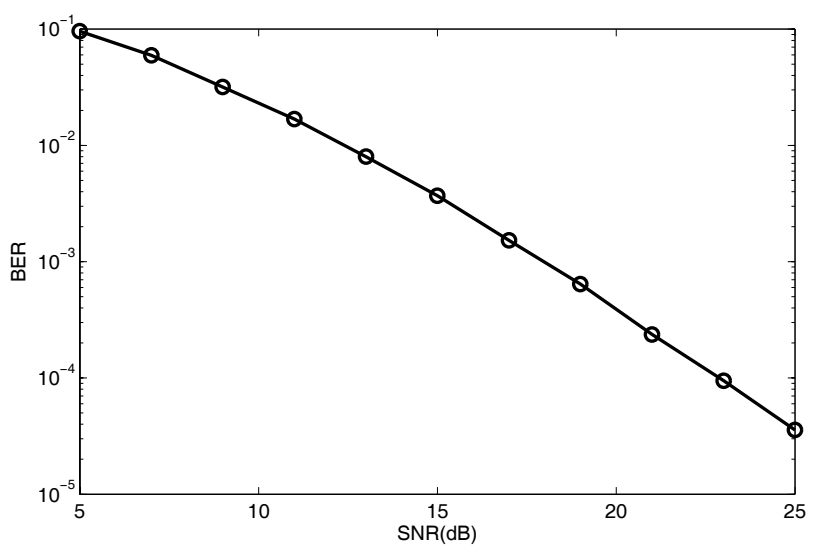

Fig. 2. BER performance of the array processing method. Results are obtained from MATLAB simulations.

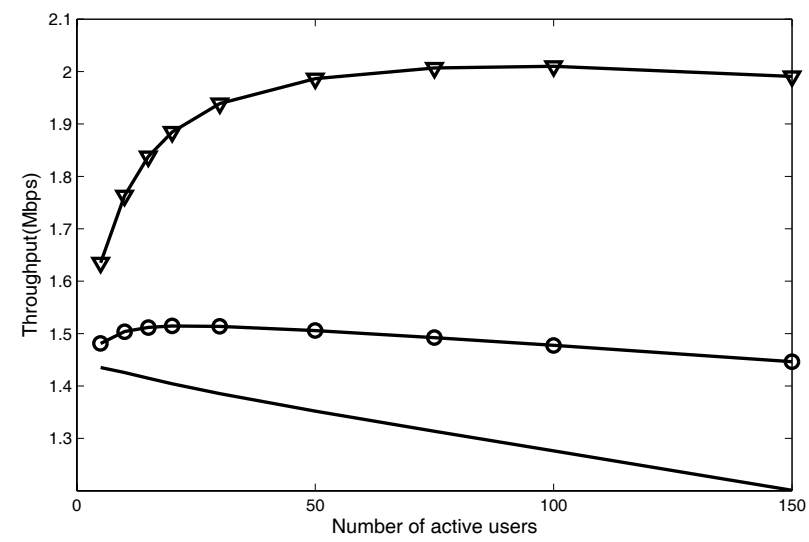

Fig. 3. A throughput comparison of the array processing MPR, the singleantenna MPR method of [2], and IEEE 802.11.

scenario for 300 seconds of simulation time. As illustrated by Fig. 1 (a), the results of simulation match the analytical results. From Fig. 1 (a), it is clear that the double-packet detector MAC enhances the throughput performance of the network.

By means of comparing simulation and analysis results for a random topology, Fig. 1 (b) validates the accuracy of our analysis when compared to the elaborate PHY model. Since the results of simulation and analysis are close to one another and follow the same trend, the analysis results provide a good approximation of the simulation results.

In Fig. 3, the throughput of the two MPR methods are compared to that of the IEEE 802.11, for a Physical layer SDU of $1024 \mathrm{~B}$. Single- and double-packet detector MACs exhibit different behaviors when the number of competing terminals increases. While the former's throughput decreases because of a higher collision probability, the latter's throughput initially increases because of a higher probability of double-packet detection. However, the throughput of the double-packet detector MAC eventually decreases with further increases of the number of nodes. This is because having a higher number of active nodes results in a higher chance of collisions, i.e., more than two simultaneous transmissions. Although the two MPR methods deliver the same number of packets per second, the overhead of fragmentation and channel coding for single- 


$$
\begin{aligned}
P_{1} & =P_{i d l e} \\
P_{2} & =P_{t r}\left(1-P_{s 1}-P_{s 2}\right) \\
P_{3} & =P_{t r} P_{s 1}\left(1-P_{R 1}\right)+P_{t r} P_{s 2}\left(1-P_{R 2}\right)^{2} \\
P_{4} & =P_{t r} P_{s 1} P_{R 1}\left(1-P_{C}\right)+2 P_{t r} P_{s 2} P_{R 2}\left(1-P_{C}\right)\left(1-P_{R 2}\right) \\
& +P_{t r} P_{s 2} P_{R 2}^{2}\left(1-P_{C}\right)^{2} \\
P_{5} & =P_{t r} P_{s 1} P_{R 1} P_{C}\left(1-P_{D 1}\right)+2 P_{t r} P_{s 2} P_{R 2}^{2} P_{C}\left(1-P_{C}\right)\left(1-P_{D 1}\right) \\
& +P_{t r} P_{s 2}\left(P_{R 2}^{2} P_{C}^{2}\left(1-P_{D 2}\right)^{2}+2 P_{R 2} P_{C}\left(1-P_{R 2}\right)\left(1-P_{D 1}\right)\right) \\
P_{6} & =P_{t r} P_{s 1} P_{R 1} P_{C} P_{D 1}\left(1-P_{A}\right)+P_{t r} P_{s 2} P_{R 2}^{2} P_{C}^{2} P_{D 2}^{2}\left(1-P_{A}\right)^{2} \\
& +2 P_{t r} P_{s 2} P_{R 2} P_{C}\left(1-P_{A}\right)\left(P_{R 2}\left(1-P_{C}\right) P_{D 1}+\left(1-P_{R 2}\right) P_{D 1}\right) \\
& +P_{t r} P_{s 2} P_{R 2} P_{C}\left(1-P_{A}\right)\left(2 P_{R 2} P_{C}\left(1-P_{D 2}\right) P_{D 2}\right) \\
P_{7} & =P_{t r} P_{s 1} P_{R 1} P_{C} P_{D 1} P_{A} \\
P_{8} & =2 P_{t r} P_{s 2} P_{R 2}^{2} P_{C}^{2} P_{D 2} P_{A}\left(P_{D 2}\left(1-P_{A}\right)+\left(1-P_{D 2}\right)\right) \\
& +2 P_{t r} P_{s 2} P_{R 2} P_{C} P_{D 1} P_{A}\left(P_{R 2}\left(1-P_{C}\right)+\left(1-P_{R 2}\right)\right) \\
P_{9} & =P_{t r} P_{s 2} P_{R 2}^{2} P_{C}^{2} P_{D 2}^{2} P_{A}^{2}
\end{aligned}
$$

antenna MPR considerably lowers the throughput gain of this method compared to that of the array processing MPR. In lower SNRs, the situation is worse and a weak MPR method like [2] may lower the throughput of the network instead of inducing a gain. In the absence of channel coding, double transmissions may fail frequently in the DATA packet transmission phase and waste a significant bandwidth. As a result, the latter situation lowers the throughput of the network in comparison to that of the IEEE 802.11. On the other hand, the performance gain of MPR-MAC is limited due to the associated overhead when using channel coding for protecting transmitted data. In this respect, our array processing MPR method is highly reliable for transmitting multiple packets and diminishes the coding overhead.

\section{CONCLUSION}

In this paper, we proposed a new cross-layer MAC/PHY algorithm capable of resolving the collision between two packets. Our algorithm leveraged a novel PHY layer interference cancellation method for collision resolution. Further, we developed analytical models of characterizing the saturation throughput of a WLAN using our proposed MPR-MAC algorithm. Our analysis considered the bit error probability of packet transmission in both single- and double-packet detection modes. The analysis also took into account the channel coding rate and the resulting packet fragmentation overhead. The results illustrated significant enhancements in the throughput of a WLAN using our MPR method. Comparisons to another MPR method highlighted the importance of the BER performance for both MPR methods. We note that it is straightforward to extend our MAC algorithm to the case of a larger number of simultaneous transmissions using the interference cancellation methods of [6]. Currently, we are working on extending our proposed MPR-MAC algorithm to the case of ad-hoc and multi-hop networks. Our future work also includes addressing the cross-layer routing schemes utilizing MPR-MAC algorithm.

\section{REFERENCES}

[1] IEEE 802.11 Standard - Part 11: Wireless LAN Medium Access Control (MAC) and Physical Layer (PHY) Specifications, Std., Jan. 2007.

[2] P. X. Zheng, Y. J. Zhang, and S. C. Liew, "Multipacket reception in wireless local area networks," in Proc. IEEE ICC '06, vol. 8, pp. 36703675, June 2006.

[3] J. J. Garcia-Luna-Aceves, H. R. Sadjadpour, and Z. Wang, "Challenges: towards truly scalable ad hoc networks," pp. 207-214, Sep. 2007.

[4] P. Wolniansky, G. Foschini, G. Golden, and R. Valenzuela, "V-BLAST: an architecture for realizing very high data rates over the rich-scattering wireless channel," in Proc. ISSSE-98, pp. 295-300, Sep. 1998.

[5] A. Naguib, N. Seshadri, and A. Calderbank, "Applications of spacetime block codes and interference suppression for high capacity and high data rate wireless systems," in Proc. 32nd Asilomar Conf. Signals, Syst. Comput., vol. 2, pp. 1803-1810, Nov. 1998.

[6] J. Kazemitabar and H. Jafarkhani, "Multiuser interference cancellation and detection for users with more than two transmit antennas," IEEE Trans. Commun., vol. 56, pp. 574-583, Apr. 2008.

[7] P. Casari, M. Levorato, and M. Zorzi, "MAC/PHY crosslayer design of MIMO ad hoc networks with layered multiuser detection," IEEE Trans. Wireless Commun., vol. 7, pp. 4596-4607, Nov. 2008.

[8] W. L. Huang, K. Letaief, and Y. J. Zhang, "Cross-layer multi-packet reception based medium access control and resource allocation for space-time coded MIMO/OFDM," IEEE Trans. Wireless Commun., vol. 7, pp. 3372-3384, Sep. 2008.

[9] H. Guo, H. Hu, and Y. Zhang, "A high-throughput random access protocol for multiuser MIMO systems," EURASIP Research Lett. Commun., pp. 1-5, 2008.

[10] S. Talwar, M. Viberg, and A. Paulraj, "Blind separation of synchronous co-channel digital signals using an antenna array-I: algorithms," IEEE Trans. Signal Process., vol. 44, pp. 1184-1197, May 1996.

[11] S. Alamouti, "A simple transmit diversity technique for wireless communications," IEEE J. Sel. Areas Commun., vol. 16, pp. 1451-1458, Oct. 1998.

[12] S. Lin and D. J. Costello, Error Control Coding, 2nd edition. PrenticeHall Inc., 2004.

[13] G. Bianchi, "Performance analysis of the IEEE 802.11 distributed coordination function," IEEE J. Sel. Areas Commun., vol. 18, pp. 535547, Mar. 2000

[14] X. Dong and P. Varaiya, "Saturation throughput analysis of IEEE 802.11 wireless LANs for a lossy channel," IEEE Commun. Lett., vol. 9, pp. 100-102, Feb. 2005.

[15] S. Sirianunpiboon, A. Calderbank, and S. Howard, "Bayesian analysis of interference cancellation for Alamouti multiplexing," IEEE Trans. Inf. Theory,, vol. 54, no. 10, pp. 4755-4761, Oct. 2008. 